\title{
Ab-Initio Modeling of Point Defects, Impurities and Diffusion in Silicon
}

\author{
Wolfgang Windl
}

Department of Materials Science and Engineering, The Ohio state University, Columbus, Ohio 43210-1178, USA

We have revisited the first-principles calculation of the diffusion coefficient for nitrogen monomers and dimers, both within the dissociative and the direct pair-diffusion picture, using densityfunctional theory and harmonic transition-state theory. From our current understanding, it seems that the results for the dissociative mechanism are in better agreement with experiment than those for the direct pair-diffusion mechanism. Based on a monomer diffusion coefficient of $D_{\mathrm{N}}(T)=7.3 \times 10^{-3} \exp \left(-0.53 \mathrm{eV} /\left(k_{B} T\right)\right) \mathrm{cm}^{2} / \mathrm{s}$, we calculate for the dissociation diffusion parameter of the dimer $P=1.6 \times 10^{9} \exp \left(-2.4 \mathrm{eV} /\left(k_{B} T\right)\right) \sqrt{\mathrm{cm}} \cdot \mathrm{s}$.

\section{Introduction}

Nitrogen doping of silicon is important because of its effects on the formation of extended defects in silicon. These include suppression of void formation $(1,2)$, increase of the mechanical strength of silicon by locking dislocations (3) and, when implanted with a sufficient dose, reduction of the oxidation rate (4), partially due to the fact that nitrogen readily binds to vacancies and can also attract oxygen $(5,6)$.

As early as the investigations of Stein (7) in 1985 it was concluded from isotope shifts that the nitrogen dimer configuration should dominate at room temperature. Jones et al. (8) confirmed this conclusion using a combination of spectroscopic and ab-initio investigations. Except for the work of Gali et al. (9), who calculated a binding energy of $1.73 \mathrm{eV}$, all theoretical investigations agree on a rather high binding energy between 3.67 and $4.3 \mathrm{eV}(6,10-12)$.

In contrast, less consensus exists about the primary mechanism of nitrogen diffusion in general and of the nitrogen pair in particular. In a variety of experimental investigations based on the out-diffusion of nitrogen from doped substrates (13) or on the indiffusion of nitrogen from the ambient (14-17) the profiles obtained were interpreted in terms of diffusion of $\mathrm{N}_{2}$ as an entity. The diffusion data obtained have been described in (18) by a diffusion constant of $D_{\mathrm{N} 2}(T)=35 \exp \left[-2.34 \mathrm{eV} /\left(k_{B} T\right)\right] \mathrm{cm}^{2} / \mathrm{s}$, with the $90 \%$ confidence interval for the activation energy ranging from 2.01 to $2.77 \mathrm{eV}$.

In the modeling analysis of Adam et al. (19), a nitrogen kick-out mechanism and binding to vacancies was assumed. Nitrogen dimers were not taken into consideration nor apparently needed to obtain good agreement with experimental profiles. A later analysis of Voronkov and Falster (20) was again based on nitrogen dimers as the prevalent defect, but it was concluded that they would diffuse via dissociation and diffusion of the monomers rather than as an entity.

Concerning atomistic modeling within first-principles methods, Schultz and Nelson calculated the migration barrier for (interstitial) nitrogen monomers to be $0.4 \mathrm{eV}$ for a split-interstitial configuration, resulting in very fast diffusion (21). Concerning nitrogen pair diffusion, Sawada et al. (22) reported values for the activation barrier of 3.3 and 
$2.8 \mathrm{eV}$ for the simultaneous and sequential movements of the nitrogen atoms, respectively. Their lower value for the energy barrier (which was found for a negative charge state) is fairly consistent with early data from Itoh and Abe (13), but outside of the confidence range given in (18). Stoddard et al. (18) calculated both the energy barrier and the diffusion prefactor for (interstitial) nitrogen monomer $(\mathrm{N})$ and $\operatorname{dimer}\left(\mathrm{N}_{2}\right)$ diffusion and found barriers of $E_{N}=0.44 \mathrm{eV}$ and $E_{\mathrm{N} 2}=2.37 \mathrm{eV}$ as well as prefactors of $D_{\mathrm{N}}=1.7 \mathrm{~cm}^{2} / \mathrm{s}$ and $D_{\mathrm{N} 2}=67 \mathrm{~cm}^{2} / \mathrm{s}$, respectively. With that, they suggested that direct pair diffusion would be the dominating diffusion mechanism. Finally, Fujita et al. (23) reported a diffusion barrier of $2.69 \mathrm{eV}$ for pair diffusion, also introducing a new intermediate metastable structure that had not been discussed previously. In the following, we will reexamine these atomistic results and reinterpret their findings.

\section{Theory of Nitrogen Diffusion}

Following Vineyard's suggestion derived from harmonic transition state theory $(24,25)$, the diffusion coefficient for a system transitioning from initial state $i$ through a transition state (saddle point) $s$ to a final state is given by

$$
D=\frac{p \cdot d^{2}}{12} v \exp \left(-\frac{\Delta S}{k_{B}}\right) \exp \left(-\frac{\Delta E}{k_{B} T}\right)
$$

where $d$ is the jump distance, $p$ is the multiplicity of jump paths, $\Delta S$ is the migration entropy (difference between saddle point and initial minimum), $\Delta E$ is the energy barrier, and $v$ is the attempt frequency. Within harmonic transition-state theory, the attempt frequency is calculated as the ratio of the product of the $\Gamma$-point vibrational frequencies of the ground state supercell over the product of all real $\Gamma$-point frequencies of the saddle point cell,

$$
v=\frac{\prod_{j=1}^{N} v_{j}^{i}}{\prod_{j=1}^{N-1} v_{j}^{s}} .
$$

The entropy of configuration is given by the natural log of the number of possible defect configurations. Within the quasiharmonic approximation, the vibrational entropy $S$ of a configuration with $N$ atoms can be calculated by $(24,25)$

$$
S=k_{B} \sum_{i=1}^{3 N}\left\{\frac{h v_{i}}{2 k_{B} T} \operatorname{coth}\left(\frac{h v_{i}}{2 k_{B} T}\right)-\ln \left[2 \sinh \left(\frac{h v_{i}}{2 k_{B} T}\right)\right]\right\} .
$$

To determine the transition state, the nudged-elastic band method (26) can be used. Among many other programs, it is implemented into VASP (27), which also can calculate the $\Gamma$-point phonon frequencies as done in (18). 


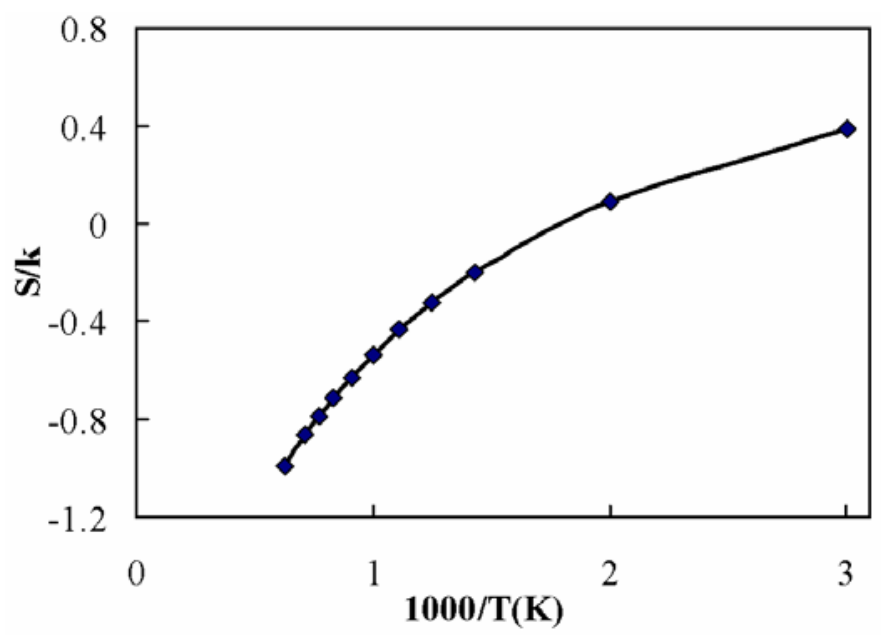

Figure 1. Migration entropy for diffusion of the nitrogen monomer as a function of temperature.

\section{Diffusion of Nitrogen Monomers}

In (18), we had found as ground state for the nitrogen monomer a bond-centered interstitial with a migration barrier of $0.44 \mathrm{eV}$. Also, since the temperature dependence of the entropy (Eq. [3]) did not completely cancel out, it had some effect on the effective migration barrier (18). Fitting the total diffusion coefficient in the temperature range from $800-1600 \mathrm{~K}$, we had found an effective diffusion coefficient of $D_{\mathrm{N}}(T)=1.7 \exp \left[-0.56 \mathrm{eV} /\left(k_{B} T\right)\right] \mathrm{cm}^{2} / \mathrm{s}$. Repeating the calculations independently with improved accuracy and reliability, we find now a different result for the prefactor, while the diffusion barrier remains unchanged. For the attempt frequency, we find

$$
v=\frac{\prod_{j=1}^{N} v_{j}^{i}}{\prod_{j=1}^{N-1} v_{j}^{s}}=\frac{2.5 \times 10^{180}}{1.1 \times 10^{179}}=24 \mathrm{THz} .
$$

The migration entropy from the difference in vibrational entropy between saddle point and initial valley, where the entropy is calculated from Eq. [3] as a function of temperature is shown in Fig. 1. With that, we fit now in the range between 800 and $1600 \mathrm{~K}$

$$
D_{\mathrm{N}}(T)=7.3 \times 10^{-3} \exp \left(-\frac{0.53 \mathrm{eV}}{k_{B} T}\right) \frac{\mathrm{cm}^{2}}{\mathrm{~s}} .
$$

Assuming that the nonlinearity in the observed nitrogen incorporation from the melt is due to an increased dimer concentration, Voronkov and Falster (28) estimated the monomer diffusivity to be approximately $D_{\mathrm{N}}(T)=1.1 \times 10^{-3} \exp \left[-0.5 \mathrm{eV} /\left(k_{B} T\right)\right] \mathrm{cm}^{2} / \mathrm{s}$, which is in good agreement with our result from Eq. [5].

\section{Diffusion of Nitrogen Dimers - Dissociative Mechanism}

Following Voronkov and Falster (28), the concentrations of monomeric (N) and dimeric $\left(\mathrm{N}_{2}\right)$ interstitial nitrogen are related by a mass action law, 


$$
\frac{C_{\mathrm{N} i}^{2}}{C_{\mathrm{N} 2}}=K,
$$

where $K$ is the equilibrium dissociation constant and is given by

$$
K=K_{0} \exp \left(-\frac{E_{b}\left(\mathrm{~N}_{2}\right)}{k_{B} T}\right)
$$

$E_{b}\left(\mathrm{~N}_{2}\right)$ is the binding energy of the two monomers into a dimer, and the prefactor $K_{0}$ is generally assumed to be close to the lattice site density $C_{\mathrm{Si}}=5 \times 10^{22} \mathrm{~cm}^{-3}$. Assuming that only monomers and dimers exist in a sample; that only the monomers are mobile; and that monomers and dimers maintain equilibrium (i.e., that $\partial C_{\mathrm{N} 2} / \partial t \approx 0$ ), the change in total nitrogen concentration is only controlled by the diffusion of the monomer (28),

$$
\frac{\partial C_{\mathrm{Ntot}}}{\partial t}=\frac{\partial\left(C_{\mathrm{N}}+2 C_{\mathrm{N} 2}\right)}{\partial t}=D_{\mathrm{N}} \nabla^{2} C_{\mathrm{N}} \text {. }
$$

As described in the introduction, the $\mathrm{N}_{2}$ binding energy used in Eq. [7] has been calculated in nearly all investigations to be at least $3.7 \mathrm{eV}(6,10-12)$. With that, at a conventional doping level with $C_{\mathrm{Ntot}}>10^{14} \mathrm{~cm}^{-3}$, Eq. [6] predicts that the dimer concentration is always significantly larger (by orders of magnitude) than the monomer concentration. With that (i.e., $C_{\mathrm{N}}<<C_{\mathrm{N} 2}$ ), Eq. [6] can be used to eliminate $C_{\mathrm{N}}$ from Eq. [8] to obtain

$$
\frac{\partial C_{\mathrm{Ntot}}}{\partial t}=D_{\mathrm{N}} \sqrt{\frac{K}{C_{\mathrm{Ntot}}}} \nabla^{2} C_{\mathrm{Ntot}}
$$

Comparing to the standard Fickian form, an effective diffusion parameter $D_{\text {eff }}$ can be defined by

$$
D_{\text {eff }}=D_{\mathrm{N}} \sqrt{\frac{K}{C_{\text {Ntot }}}}
$$

To get rid of the concentration dependence, one can also define a "dissociation diffusion parameter" $P(28)$,

$$
D_{\text {eff }}=\frac{P}{\sqrt{C_{\mathrm{Ntot}}}}, \quad P=D_{\mathrm{N}} \sqrt{K}
$$

Using the standard Arrhenius form for the monomer diffusion coefficient with prefactor $D_{\mathrm{N}}^{0}$ and activation energy $E_{\mathrm{N}}^{\mathrm{m}}$ in combination with Eq. [7], we find

$$
P=D_{\mathrm{N}}^{0} \sqrt{C_{\mathrm{Si}}} \exp \left(-\frac{E_{b}\left(\mathrm{~N}_{2}\right) / 2+E_{\mathrm{N}}^{\mathrm{m}}}{k_{B} T}\right) \equiv P_{0} \exp \left(-\frac{E_{P}}{k_{B} T}\right)
$$




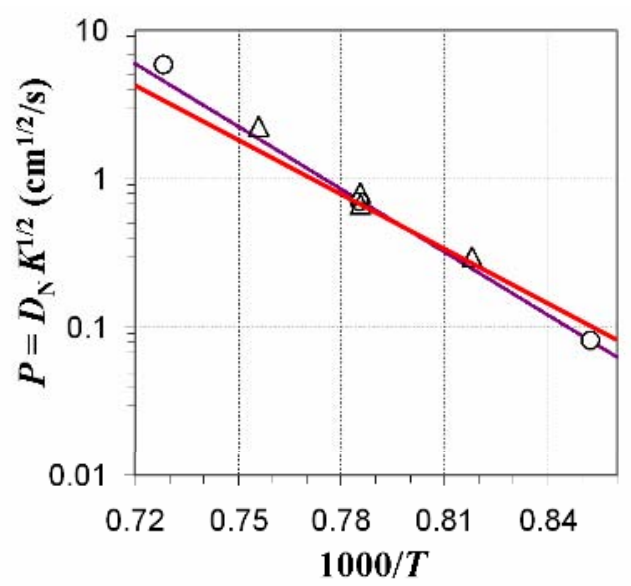

Figure 2. Dissociation diffusion parameter P.

In agreement with previous work, we calculate the binding energy of the dimer over two monomers as $3.7 \mathrm{eV}$. In combination with the diffusivity from Eq. [5], we thus find

$$
P=1.6 \times 10^{9} \exp \left(-\frac{2.4 \mathrm{eV}}{k_{B} T}\right) \sqrt{\mathrm{cm}} \cdot \mathrm{s} .
$$

This result can now be compared to values from a fit to experimental data, where Voronkov and Falster have determined $P_{0}=8.4 \times 10^{10} \sqrt{\mathrm{cm}} \cdot \mathrm{s}$ and $E_{P}=2.8 \mathrm{eV}$ (28) and $P_{0}=4 \times 10^{10} \sqrt{\mathrm{cm}} \cdot \mathrm{s}$ and $E_{P}=2.7 \mathrm{eV}(29)$, respectively. Figure 2 shows our results in comparison to the measured points and the fitted curve from (29), where we find excellent agreement between theory and experiment. This strongly supports Voronkov and Falster's suggestion of a dissociative mechanism for $\mathrm{N}_{2}$ diffusion.

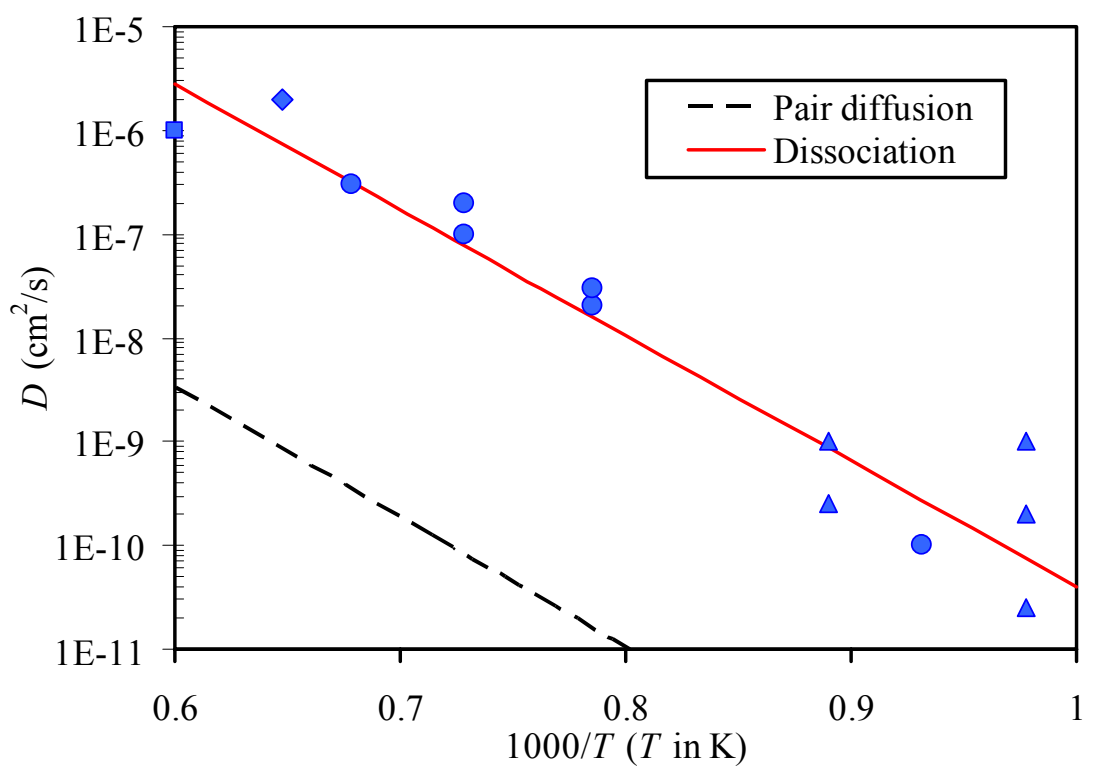

Figure 3. Calculated diffusion coefficient for $\mathrm{N}_{2}$ diffusion, using the direct pair-diffusion mechanism (black dashed line) and the dissociative mechanism with a nitrogen concentration $C_{\text {Ntot }}$ of $10^{15} \mathrm{~cm}^{-3}$ (red solid line), in comparison to experimental values [circles from (12); diamonds from (13); squares from (14); triangles from (15) and (16)]. 


\section{Diffusion of Nitrogen Dimers - Pair-Diffusion Mechanism}

Assuming that the barrier of $2.37 \mathrm{eV}$ had been determined correctly in (18) for the direct pair-diffusion mechanism (which, however, is small compared to other calculations as discussed below), we recalculate with improved accuracy and care the corresponding prefactor and find a value of $5 \times 10^{-2} \mathrm{~cm}^{2} / \mathrm{s}$ instead of the previously $67 \mathrm{~cm}^{2} / \mathrm{s}$. This makes the pair-diffusion mechanism three orders of magnitude slower than experiment (Fig. 3).

Looking at the other calculations of the diffusion parameters for the pair diffusion mechanism, we find work by Sawada et al. (21) and Fujita et al. (23), where the former concludes a minimum pair diffusion barrier of $2.8 \mathrm{eV}$, whereas the latter reports $2.69 \mathrm{eV}$. Nevertheless, both find the so-called Humble configuration as an intermediate metastable configuration, with symmetric minimum-energy paths between initial and Humble; and final and Humble configurations, respectively. We re-plot both data sets along with a spline interpolation in Fig. 4.

As already mentioned in (23), (21) did not try to spline-interpolate their data, resulting in a lower energy barrier than one would get by the usual spline interpolation. With that, we find for the neutral (negative) charge-state data from (21) an energy barrier of $3.05 \mathrm{eV}(2.90 \mathrm{eV})$. Due to the band-gap problem in standard DFT calculations, there is probably a larger error bar associated with the barrier for the negative charge state, which, if it was corrected, should be expected to be higher (23).

In contrast to (21), (23) finds an intermediate minimum configuration between initial and Humble configurations, which they label low symmetry (LS) configuration and which has a formation energy of approximately $1.85 \mathrm{eV}$ with respect to the initial nitrogen-pair configuration. The barrier between initial and LS configurations is reported to be $2.69 \mathrm{eV}$, and the second barrier between LS and Humble configurations is $1.19 \mathrm{eV}$ (23). Our spline interpolation of the original data finds identical values. (23) then argues that the first energy barrier of $2.69 \mathrm{eV}$ would limit the overall diffusion, since it was considerably higher than the second one. However, following standard transition state theory (31), the effective barrier would not be expected to be the highest intermediate barrier, but the maximum overall barrier with respect to the initial state, which in this case is the barrier between the LS and the Humble configurations, which has a value of $3.04 \mathrm{eV}$ with respect to the initial state. Thus, besides the fact that (23) finds the additional intermedi-
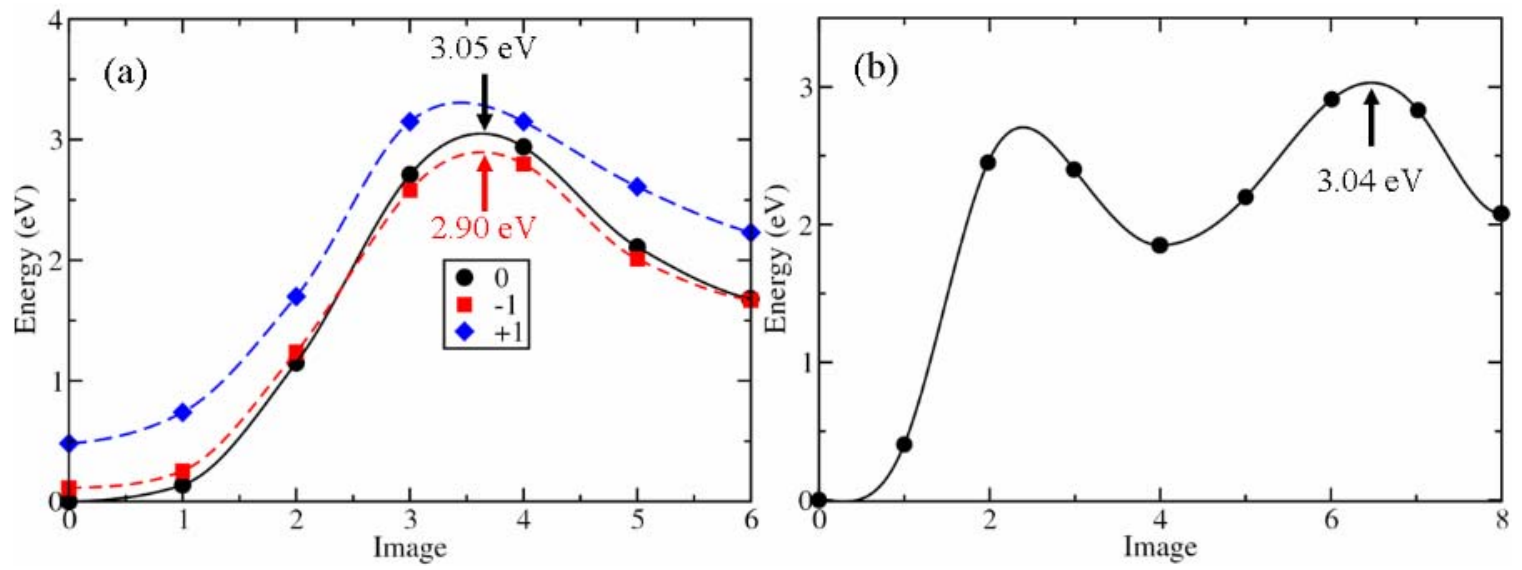

Figure 4. Minimum energy paths for nitrogen pair diffusion from the initial to the intermediate Humble configuration, redrawn after previous work. The points are data taken from (a) (21) and (b) (23), respectively [(21) has data for different charge states as indicated in (a)]. The lines are Akima spline interpolations of the original data. The maxima of the curves are indicated by arrows along with their energy values. 
ate metastable LS state, they agree very well on the (neutral) barrier of just above $3 \mathrm{eV}$. This barrier is higher than the value of $2.34 \mathrm{eV}$ fitted to a compilation of experimental data in (18) and is also outside of the $90 \%$ confidence interval for the activation energy ranging from 2.01 to $2.77 \mathrm{eV}$. It is, however, somewhat closer to the recent values of 2.7-2.8 eV determined by Voronkov and Falster for the dissociation mechanism $(28,29)$.Nevertheless, unless the prefactor for the $\sim 3 \mathrm{eV}$ saddle points is in the range of $10^{4} \mathrm{~cm}^{2} / \mathrm{s}$, orders of magnitude larger than the value of $5 \times 10^{-2} \mathrm{~cm}^{2} / \mathrm{s}$ calculated above for the saddle point from (18), the pair-diffusion mechanism will be negligible in comparison to the dissociation mechanism. In order to check this, we calculated the prefactors within the quasiharmonic approximation described above for the (approximate) saddle point configurations reported in Refs. (21) and (23). We find them to be on the order of $10^{-2} \mathrm{~cm}^{-2} / \mathrm{s}$, giving strong support that the dissociative mechanism may indeed be the dominating one.

\section{Conclusions}

We have revisited the calculation of the diffusion coefficient for nitrogen, both within the dissociative and the direct pair-diffusion picture, using density-functional theory and harmonic transition-state theory. From our current results, it seems that the results for the dissociative mechanism are in better agreement with experiment than the direct pairdiffusion mechanism. In more detail, for the dissociation diffusion parameter, we calculate $P=1.6 \times 10^{9} \exp \left(-2.4 \mathrm{eV} /\left(k_{B} T\right)\right) \sqrt{\mathrm{cm}} \cdot \mathrm{s}$, based on a monomer diffusion coefficient of $D_{\mathrm{N}}(T)=7.3 \times 10^{-3} \exp \left(-0.53 \mathrm{eV} /\left(k_{B} T\right)\right) \mathrm{cm}^{2} / \mathrm{s}$, which compares favorably with experimentally determined values of $P=8.4 \times 10^{10} \exp \left(-2.8 \mathrm{eV} /\left(k_{B} T\right)\right) \sqrt{\mathrm{cm}} \cdot \mathrm{s} \quad$ (28) and $P=4 \times 10^{9} \exp \left(-2.4 \mathrm{eV} /\left(k_{B} T\right)\right) \sqrt{\mathrm{cm}} \cdot \mathrm{s}$ (29). On the other hand, revisiting previous work $(18,21,23)$ on dimer diffusion, we conclude that direct pair diffusion should be more than three orders of magnitude smaller than diffusion by dissociation, making its overall contribution to nitrogen diffusion negligible.

\section{Acknowledgments}

We would like to thank Naomi Fujita for fruitful discussions stimulating the new work presented in this paper. This work has been supported by the National Science Foundation under contract number DMR-0244724 and DMR-0820414. We also thank the Ohio Supercomputer Center for computational time under project number PAS0072.

\section{References}

1. T. Abe, H. Harada, and J. Chikawa, in Defects in Semiconductors II, S. Mahajan and J. W. Corbett, Editors, PV 14, p. 1, Materials Research Society Proceedings Series, Pittsburgh, PA (1983).

2. K. Nakai, Y. Inoue, H. Yokota, A. Ikari, J. Takahashi, A. Tachikawa, K. Kitahara, Y. Ohta, W. Ohashi, J Appl. Phys., 89, 4301 (2001).

3. K. Sumino, I. Yonenaga, M. Imai, T. Abe, J. Appl. Phys., 54, 5016 (1983).

4. W. J. M. J. Josquin, Y. Tamminga, J. Electrochem. Soc., 129, 1803 (1982).

5. A. Karoui, F. S. Karoui, G. Rozgonyi, M. Hourai, and K. Sueoka, J. Electrochem. Soc., 150 G771 (2003). 
6. H. Kageshima, A. Taguchi, and K. Wada, Appl. Phys. Lett., 76, 3718 (2000).

7. H. J. Stein, in Thirteenth International Conference on Defects in Semiconductors, L. C. Kimerling and J. M. Parsey, Jr., Editors, p. 839, The Metallurgical Society of AIME (1985).

8. R. Jones, S. Öberg, F. Berg Rasmussen and B. Bech Nielsen, Phys. Rev. Lett., 72, 1882 (1994).

9. A. Gali, J. Miro, P. Deák, C. P. Ewels, R. Jones, J. Phys.: Condens. Matter, 8, 7711 (1996).

10. H. Sawada and K. Kawakami, Phys. Rev. B, 62, 1851 (2000).

11. A. Karoui, F. Sahtout Karoui, G. A. Rozgonyi, M. Hourai, and K. Sueoka, in Semiconductor Silicon 2002, H. R. Huff et al., Editors, PV2002-2, p. 670, The Electrochemical Society Proceedings Series, Pennington, NJ (2002).

12. J. P. Goss, I. Hahn, and R. Jones, Phys. Rev. B, 67, 045206 (2003).

13. T. Itoh and T. Abe. Appl. Phys. Lett., 53, 39 (1988).

14. A. Hara, T. Fukuda, T. Miyabo, and I. Hirai, Appl. Phys. Lett., 54, 626 (1989).

15. G. J. Willems and H. E. Maes, J. Appl. Phys., 73, 3256 (1993).

16. N. Fuma, K. Tashiro, K. Kakumoto, and Y. Takano, Mat. Sci. Forum, 196-201, 797 (1995).

17. N. Fuma, K. Tashiro, K. Kakumoto, and Y. Takano, Jpn. J. Appl. Phys., Part 1, 35, 1993 (1996).

18. N. G. Stoddard, P. Pichler, G. Duscher, and W. Windl, Phys. Rev. Lett., 95, 025901 (2005).

19. L. S. Adam, M. E. Law, O. Dokumaci, and S. Hegde, J. Appl. Phys., 91, 1894 (2002).

20. V. Voronkov and R. Falster, Sol. State Phenom., 95-96, 83 (2004).

21. P. A. Schultz and J. S. Nelson, Appl. Phys. Lett., 78, 736 (2001).

22. H. Sawada, K. Kawakami, A. Ikari and W. Ohashi, Phys. Rev. B, 65, 075201 (2002).

23. N. Fujita, R. Jones, J. P. Goss, T. Frauenheim, S. Öberg, and P. R. Briddon, Solid State Phenom., 108-109, 407 (2005).

24. D. Chandler, Introduction to Modern Statistical Mechanics, p. 70, Oxford University Press, New York (1987).

25. R. LeSar, R. Najafabadi, and D. J. Srolovitz, Phys. Rev. Lett., 63, 624 (1989).

26. H. Jónsson, G. Mills, and K. W. Jacobsen, in Classical and Quantum Dynamics in Condensed Phase Simulations, B. J. Berne, G. Ciccotti, and D. F. Coker, Editors, p. 385, World Scientific, Singapore (1998).

27. G. Kresse and J. Hafner, Phys. Rev. B, 47, 58 (1993); Phys Rev. B, 49, 14251 (1994). G. Kresse and J. Furthmüller, Comput. Mater. Sci., 6, 16 (1996); Phys. Rev. B, 55, 11169 (1996).

28. V. V. Voronkov and R. Falster, J. Appl. Phys., 100, 083511 (2006).

29. V. V. Voronkov and R. Falster, ECS Trans., 4, 113 (2006).

30. W. Windl, Phys. Stat. Sol. B, 241, 2313 (2004).

31. P. Pechukas, in Dynamics of Molecular Collisions, Part B, Modern Theoretical Chemistry, vol. 2, W. H. Miller, Editor, p. 269, Plenum, New York (1976). 